\title{
BMJ Open Mental health informed physical activity for first responders and their support partner: a protocol for a stepped-wedge evaluation of an online, codesigned intervention
}

\author{
Grace McKeon, ${ }^{1}$ Zachary Steel, ${ }^{1,2,3}$ Ruth Wells, ${ }^{1}$ Jill M Newby, ${ }^{4}$ \\ Dusan Hadzi-Pavlovic, ${ }^{1}$ Davy Vancampfort, ${ }^{5,6}$ Simon Rosenbaum ${ }^{1,3}$
}

To cite: McKeon G, Steel Z, Wells R, et al. Mental health informed physical activity for first responders and their support partner: a protocol for a stepped-wedge evaluation of an online, codesigned intervention. BMJ Open 2019;9:e030668. doi:10.1136/ bmjopen-2019-030668

- Prepublication history and additional material for this paper are available online. To view these files, please visit the journal online (http://dx.doi org/10.1136/bmjopen-2019030668).

Received 26 March 2019 Revised 06 August 2019 Accepted 09 August 2019

Check for updates

(C) Author(s) (or their employer(s)) 2019. Re-use permitted under CC BY-NC. No commercial re-use. See rights and permissions. Published by BMJ.

For numbered affiliations see end of article.

Correspondence to

Grace McKeon;

g.mckeon@unsw.edu.au

\section{ABSTRACT}

Background First responders (police, fire and ambulance officers) are at a significantly increased risk of experiencing poor mental health, including depression and post-traumatic stress disorder. These conditions are associated with high rates of cardiovascular disease, in part due to low levels of physical activity (PA) and high levels of sedentary behaviour. Using a person's social support system may be an effective solution to help increase PA levels to improve mental and physical health outcomes. We will examine the efficacy of a group-based online intervention in increasing PA in first responders and their support partners, iteratively codesigned with advisors with lived experience of mental illness among first responders.

Methods This study will recruit a convenience sample of self-identified sedentary first responders and their selfselected support partners to a 10-week PA programme delivered through a private Facebook group. We will deliver education on predetermined topics related to PA and diet and provide participants with an activity tracker (Fitbit). A stepped-wedged design will be applied to compare multiple baselines to intervention and follow-up phases within subjects. Five cohorts of $n=20$ will be recruited, with each cohort randomised to a different baseline length. Our primary outcome will be psychological distress (Kessler-6). Secondary outcomes include feasibility, selfreport and objective PA data (Simple Physical Activity Questionnaire and Fitbit accelerometry), depression and anxiety (Depression Anxiety and Stress Scale-21 items), post-traumatic stress disorder symptoms (PTSD Checklist for DSM-5), quality of life Assessment of Quality of Life-6 dimensions, sleep quality (The Pittsburgh Sleep Quality Index), suicidal ideation (Suicidal Ideation Attributes Scale) and social support for exercise. The mobile data collection platform MetricWire will be used.

Ethics and dissemination Ethical approval was obtained from the University of New South Wales, Deupty ViceChancellor Research, Human Research Ethics Committee on 3 June 2019, HC180561. Findings will be published in peer-reviewed journals and disseminated at national conferences.

Trial registration number ACTRN12619000877189.
Strengths and limitations of this study

- The novel online delivery offers a highly scalable and potentially cost-effective solution to engaging first responders, particularly those in rural and remote settings.

- The inclusion of support partners in the study is intended to increase motivation and improve the physical and mental health of both participants.

- The stepped-wedge design replaces the need for a control group by making each participant act as their own comparison prior to implementation.

- The short follow-up period will fail to capture any long-term benefits of the intervention.

- Recruitment though Facebook may introduce a bias in regard to the population sample.

\section{INTRODUCTION}

First responders including police, fire, ambulance, rescue and other emergency services personnel are at a significantly increased risk of experiencing poor physical and mental health. Cumulative exposure to traumatic events puts first responders at a high risk of developing serious stress and psychological consequences from exposure to trauma. ${ }^{1}$ Post-traumatic stress disorder (PTSD) has been identified as one of the most notable psychological consequences from repeated exposure to trauma. ${ }^{23} \mathrm{~A}$ recent review of international studies including over 20000 first responders concluded that the prevalence of PTSD among current first responders was 1 in $10 .{ }^{4}$ This prevalence is far in excess of the general population ${ }^{5}$ yet is still likely to be significantly underestimated, given symptom onset is often delayed in relation to exposure and rates are likely to be higher among retired workers. ${ }^{16}$ 
Regular exposure to trauma is an unavoidable consequence of the first-responder role. The need for an initial response may occur as a result of a natural disaster, an accident or a deliberate human act often involving death and/or injury and/or loss of property. ${ }^{7}$ The unpredictable nature of these emergencies results in high levels of stress and sometimes put first responder's own safety at risk. The long-term impact of traumatic stress on mental health has been identified as one of the major determinants of impairment and disability across the globe. ${ }^{8}$ Adverse consequences of PTSD include impairments in psychosocial functioning, ${ }^{9}$ substance abuse ${ }^{10}$ comorbid mental disorders $^{9}$ and an increase risk of suicidal behaviour. ${ }^{11}$ In addition to the adverse impact on an individual's mental health and well-being, PTSD has been identified as a risk factor for obesity, diabetes and metabolic syndrome. ${ }^{12-16}$ It is important to note that first responders without PTSD also show high rates of cardiovascular disease and metabolic syndrome. ${ }^{217}$

A modifiable risk factor contributing to the relationship between poor physical health and PTSD is low levels of physical activity (PA) and high levels of sedentary behaviour. People with PTSD are less likely to engage in regular PA compared with their pre-PTSD levels. ${ }^{18}$ In the past decade, interest in and evidence for exercise as a component of treatment in mental illness has significantly increased. ${ }^{19}{ }^{20} \mathrm{PA}$ and its structured subset, exercise, is an efficacious and scalable intervention that can improve both physical and mental health outcomes without risk of significant adverse effects. ${ }^{21}$ In addition, since exercise is typically viewed as non-stigmatising, it is likely to be well accepted among this population. A recent meta-analysis concluded that $33.1 \%$ of first-responders experience stigma regarding mental health care. ${ }^{22}$ The most frequently endorsed concerns were fears that seeking psychological help would negatively impact one's career and fears regarding confidentiality.

For people living with mental illness, PA has been shown to reduce symptoms of depression and anxiety, improve sleep quality and reduce alcohol dependence. ${ }^{21} 2324$ Exercise delivered in addition to usual care in patients with PTSD has been shown to reduce PTSD and depressive symptoms, improve sleep quality and reduce waist circumference. ${ }^{25} \mathrm{PA}$ also has great potential as a preventative intervention, which is crucial for groups at increased risk of psychological injuries, such as in first responders. Recent data from the Health Study of Nord-Trøndelag County study showed that as little as $60 \mathrm{~min}$ per week of additional PA could prevent $12 \%$ of all incident cases of depression. ${ }^{23}{ }^{26}$ Despite the known mental health and cardioprotective benefits of increased PA, there has not been extensive research exploring ways to engage first responders in preventative or treatment-based exercise.

Delivering PA interventions to Australia's geographically dispersed first-responder population creates major logistical challenges. As noted in recent expert guidelines on the diagnosis and treatment of PTSD in first responders, the emergence of e-health interventions via the internet or mobile devices offers the potential to increase access to evidence based interventions for first responders. ${ }^{2}$ Facebook groups have previously been used effectively to deliver behavioural change interventions in both the general population and in people with serious mental illness. ${ }^{27} 28 \mathrm{~A}$ recent review found that eHealth technologies including social media platforms such as Facebook can increase PA levels and may have beneficial effects on mental health outcomes in patients with mental illness. ${ }^{29}$ The reach and popularity of social networking sites such as Facebook may therefore offer highly scalable and cost-effective opportunities for engaging first responders in PA-focused lifestyle interventions.

To best develop interventions and ensure routine translation into real-world settings, the voices of those who have lived through the experience of PTSD or its triggers in the course of their duties are required. Recent codesign recommendations from consumers experiencing mental health problems and exercise practitioners to inform effective PA interventions included social support, person-centred options, connection and a sense of belonging and access to information and education. ${ }^{30}$ With this in mind, our study puts forward a novel approach from a service design perspective in an effort to maximise and tailor future PA participation for first responders.

The codevelopment of our intervention with lived experience personnel identified the importance of facilitating social support. Previous research has found that social isolation is a significant contributing factor to inactivity and that social support for people with mental illness is positively associated with increased PA. ${ }^{17} 31$ Bolstering existing support by including a support partner may therefore be an effective solution to increasing the potency and long-term effectiveness of lifestyle interventions. ${ }^{32}$

To address recent calls regarding the need for novel, innovative approaches to address the issue of mental illness among first responders, we have developed a pilottested PA programme. In October 2018, we ran a pilot of the intervention. This pilot work demonstrated that the intervention was feasible and that first responders and their selected support partners were highly satisfied with using the Facebook group. We have consolidated our protocol based on the results and qualitative feedback. Therefore, the present large-scale study aims to determine the efficacy of the intervention on mental health symptoms and PA levels.

\section{METHODS}

\section{Study design}

This study will apply a stepped-wedge design. This involves the collection of data on multiple occasions before the intervention to detect whether the intervention has an effect significantly greater than the underlying secular trend. ${ }^{33} 34$ Consecutive observations will be interrupted by the intervention to see if the slope or level of the series changes following the intervention. The baseline lengths 


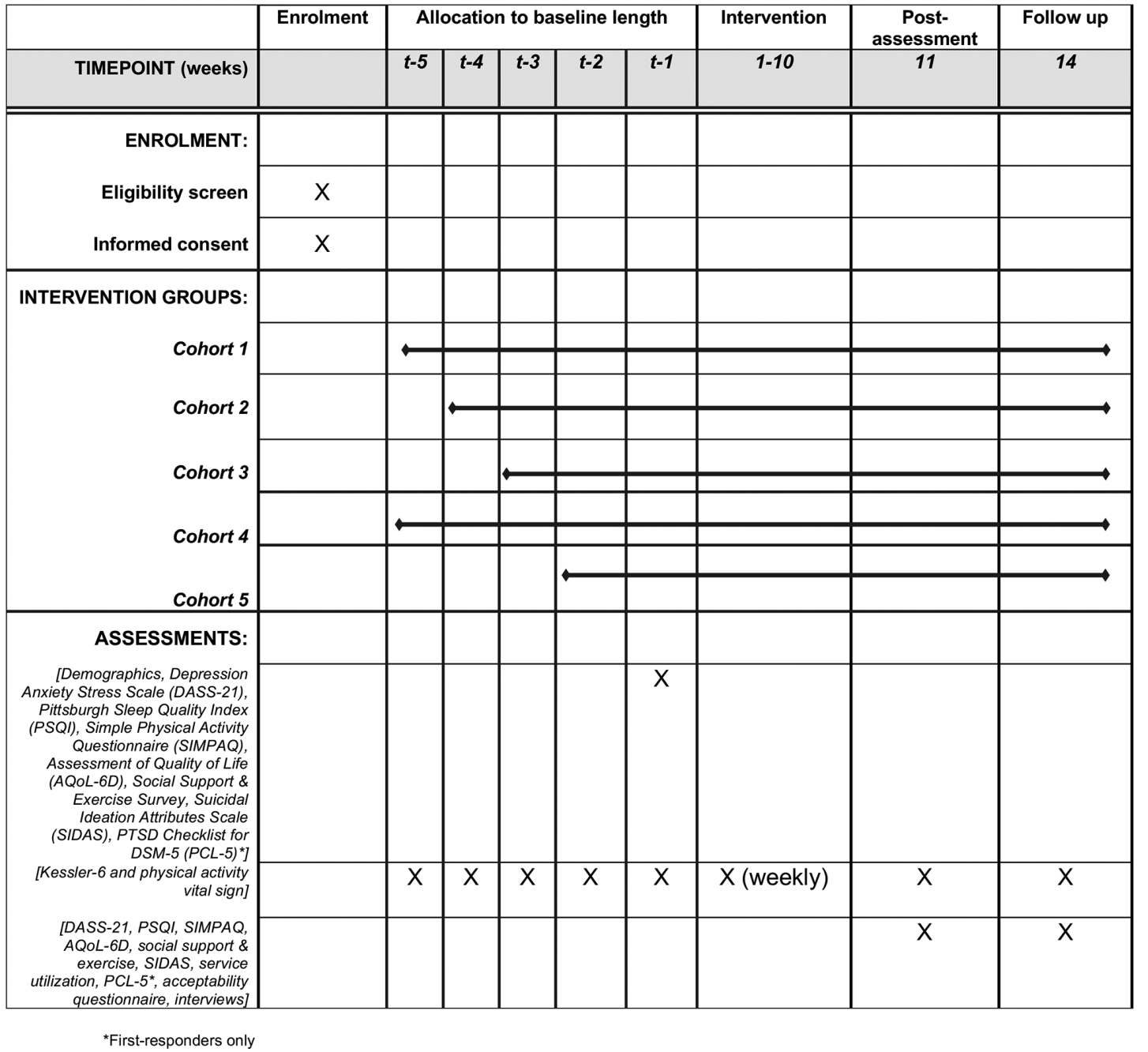

Figure 1 Schedule of enrolment, intervention and assessment.

of each group taken through the programme has been randomised between 2 weeks and 5 weeks so that treatment is introduced at different times for each cohort to control for the effects of time. A random number generator will be used in excel to generate values between 0 and 1 . This will then be used to order the length of baseline into a random order. An example of randomised baseline lengths for different cohorts and the schedule of participants through the trial are shown in figure 1 .

\section{Participants}

Participants will be recruited through Behind the Seen, a not-for-profit, community initiative that aims to increase awareness and reduce stigma towards mental health issues facing first responders and their families (http://www. behindtheseenaustralia.com). The facilitators of Behind the Seen will post the advertisement on their Facebook page, which includes information regarding the study aims, inclusion and exclusion criteria. Interested participants will contact the study investigator who will provide information about the study including the participant information statement and will be screened using self-administered questionnaires on the MetricWire application.
This study will recruit a convenience sample of former or current first responders, aged 18-65 years who are currently physically inactive according to the PA guidelines, defined as engaging in less than $150 \mathrm{~min}$ of moderate-vigorous PA per week. Five cohorts with 20 participants in each will be recruited (10 first responders and their support partners). The first cohort will begin in August 2019.

Participants must be cleared to participate in an exercise programme using the Exercise Sports Science Australia Pre-Screening Tool. ${ }^{33}$ This is a self-administered questionnaire that aims to identify individuals with a known disease, or signs or symptoms of disease who may be at a higher risk of an adverse event during exercise. Participants must also speak English, have internet access and an active Facebook account or be willing to create one. First responders will be required to nominate a support partner. This may be any person with a close personal relationship to them, for example, partner, family member, carer or friend with whom they have at least weekly in-person contact with. This person must also be between 18 years and 65 years and be medically cleared to exercise. 
Although we will not specifically target those first responders with a diagnosed mental health condition, by nature of the target population, it is expected that a high proportion of the participants will be receiving treatment for a mental disorder. Participants will be screened for psychological distress using the Kessler-6 (K6). A score of $>25$ suggests the person is likely to have a severe mental disorder. Participants who score $>25$ and who are not receiving treatment or whose medications have changed in the past 4 weeks will be excluded and referred to a local health professional. We will also screen scores in the Suicidal Ideation Attributes Scale (SIDAS). If a participant receives a score $>21$, which is indicative of a high risk of suicidal behaviour, they will be called by a psychologist who will conduct a suicide risk assessment. Based on this assessment, the psychologist will determine whether the applicant is eligible to participate in the study and refer to mental health services where appropriate.

\section{Intervention}

The PA intervention is composed of 10 weeks of content, delivered online through a private Facebook group. Both the first responders and their nominated support partners will be encouraged to be actively involved on the Facebook page and with the programme. All of the content for the current intervention has been informed based on the results and feedback from our pilot study. The research team will provide education on predetermined weekly topics and facilitate discussion boards, for example, goal setting, barriers and sedentary behaviour. Weekly content will include information, exercise demonstration videos, links to existing online resources, images, step count competitions and discussion questions. These posts will all occur from the study generated account, and the page will be checked by the facilitators at least once per day, Monday-Friday. Defined behaviour change techniques such as informing the behaviour-health link, barrier identification, general encouragement, instruction, self-monitoring, social support, specific goal setting and feedback will be incorporated into the programme.

Participants will also have the option to join a weekly group telehealth call. The facilitator will lead the discussion and provide further education on the same weekly topic as the Facebook group for approximately 20-30 min. Participants will be able to join in on the discussion and ask questions. The weekly content of the call and Facebook group is outlined in table 1 and a more comprehensive manual is located in the online supplementary appendix.

Participants will be provided with a Fitbit Inspire prior to the intervention and asked to wear it daily. This is an activity tracker worn on the wrist that measures steps and active minutes and syncs to a personal Fitbit dashboard where participants can set personal goals and track progress. The Fitbits will be provided as a way to support self-monitoring. Participants will need to activate their device and set up a Fitbit account. The research team will be using Fitbit's health solutions platform that provides access to data from the participants activity trackers. Participants will also have the option to be a part of the health solutions group where they can see each other's step counts and compete in weekly competitions. After the 10-week trial, participants will have the choice to remain in the Facebook group and continue to use it without input from facilitators. There will be a follow-up period of 1 month.

\section{Patient and public involvement}

The iterative development of our online intervention has occurred through collaboration with project advisors with lived experience of working as a first responder and of living with mental illness related to occupational trauma. The research team and the facilitators of Behind the Seen designed the manual containing the weekly content for the group. Weekly meetings will occur throughout the programme to review progress of the group and make plans for the following week based on participants' posts and engagement. The two facilitators from Behind the Seen will act as peer support members in the group, posting from their own accounts to help facilitate relatable discussion. Two to three volunteers from the pilot phase of the programme will be invited to the Facebook group to also act as peer support members. Their role will be to help deliver content and share their personal experiences with the programme, bridging the gap between participants and facilitators.

\section{OUTCOMES}

\section{Data collection}

Data will be collected from both the first responders and their support person via self-report questionnaires, Fitbit data and one-on-one Skype interviews. Data collection including demographics and all self-report questionnaires will occur through the MetricWire platform. This is a phone application that allows participants to complete research questionnaires in a safe and accessible manner.

\section{Primary outcome}

Psychological distress

The K6 is a self-report questionnaire that will be used to assess psychological distress. ${ }^{34}$ The $\mathrm{K} 6$ will be assessed every week throughout the trial including the baseline period.

\section{Secondary outcomes}

Feasibility and acceptability

Feasibility will be defined by usage of the Facebook group (manual calculation of the sum of posts, likes and comments), Fitbit compliance (\% of days worn) and participant retention (completion of questionnaires and retention in programme).

Acceptability will be assessed at postintervention using the 14-item feasibility and acceptability questionnaire that has been used previously to measure participant responses to a private Facebook group. ${ }^{35}$ Responses are answered on 


\begin{tabular}{|c|c|}
\hline Topic & Content \\
\hline Welcome & $\begin{array}{l}\text { Participants asked to introduce themselves, for example, occupation, why they joined } \\
\text { the group. } \\
\text { Instructions provided on Fitbits. }\end{array}$ \\
\hline Goal setting & $\begin{array}{l}\text { How to write a SMART goal. For example, participants encouraged to increase their } \\
\text { step count from last week by } 5 \%-10 \% \text {. } \\
\text { Participants asked to write goals (short term, long term and one with support person) } \\
\text { and post them on the Facebook group. } \\
\text { Benefits of self-monitoring and ways to do it, for example, Fitbit, training diaries. }\end{array}$ \\
\hline $\begin{array}{l}\text { Benefits of physical } \\
\text { activity }\end{array}$ & $\begin{array}{l}\text { Link between physical and mental health explained. } \\
\text { Physical and mental health benefits (eg, improved mood, sleep, decreased anxiety and } \\
\text { stress and so on). } \\
\text { Video links and fact sheets provided. }\end{array}$ \\
\hline Support & $\begin{array}{l}\text { Information provided on how to be a helpful support person (practical support, } \\
\text { effective communication and exercising together). } \\
\text { Information provided on social support for increasing motivation. }\end{array}$ \\
\hline Sedentary behaviour & $\begin{array}{l}\text { Risks associated with sedentary behaviour, for example, increased mortality risk. } \\
\text { How to increase incidental activity including ways to incorporate PA into everyday life. } \\
\text { - Minimise time spent sitting and encourage breaking up long periods of sitting. }\end{array}$ \\
\hline Aerobic exercise & $\begin{array}{l}\text { Australian guidelines. } \\
\text { Finding an exercise you enjoy. }\end{array}$ \\
\hline Resistance exercise & $\begin{array}{l}\text { Australian guidelines (strength training at least } 2 \times \text { per week). } \\
\text { Exercise safety (eg, importance of a warm up). } \\
\text { Videos of simple workouts (eg, squats and push ups against a wall). } \\
\text { The importance of progression and ways to do it, for example, using the frequency, } \\
\text { intensity, time and type principle. }\end{array}$ \\
\hline Review & $\begin{array}{l}\text { How to maintain an exercise programme. } \\
\text { Community programme discussed, for example, gyms, community runs. } \\
\text { Review of goals. } \\
\text { Celebration of progress. }\end{array}$ \\
\hline
\end{tabular}

PA, physical activity.

a 7-point Likert scale (strongly disagree-strongly agree). Participants will also be invited to participate in a $20 \mathrm{~min}$ one-on-one semistructured interview via Skype. The interview will cover topics such as likes, dislikes, effectiveness and recommendations for future iterations. Interviews will be recorded and transcribed verbatim. They will then be qualitatively analysed using thematic analysis to determine key themes identified by participants. ${ }^{36}$

Minutes of PA

PA levels will be assessed both objectively (Fitbit data) and subjectively (the PA vital sign and Simple Physical Activity Questionnaire (SIMPAQ) ). ${ }^{37}$ The PA vital sign is a clinical assessment tool designed to gauge levels of moderate to vigorous PA and takes less than $30 \mathrm{~s}$ to complete. This will be assessed weekly throughout the baseline, intervention and follow-up periods. The SIMPAQ is a fiveitem clinical tool designed to assess PA among populations at high risk of sedentary behaviour. For the purpose of this study, the SIMPAQ has been adapted into an online version using MetricWire. The SIMPAQ traditionally requires an interviewer to form simple calculations to account for hours left in the day based on previous answers, and our online version uses basic formulas to still allow participants to crosscheck responses based on how many hours they need to account for. The SIMPAQ will be used at baseline, postintervention and follow-up. Total time per week of walking, moderate-vigorous PA (MVPA), incidental and sedentary time will be assessed.

Fitbit accelerometry data will also be gathered for the 10-week intervention and 4-week follow-up. Participants 
will need to provide consent to allow the researchers to have access to their daily step count and active minutes (MVPA). The research team will be using Fitbit's health solutions platform to access the data from the participants' activity trackers.

\section{Depression and anxiety}

The Depression Anxiety and Stress Scale will be used to assess the effects of the programme on depressive and anxiety symptoms. ${ }^{39}$ The DASS21 is a 21 -item self-report instrument that measures the related negative emotional states of depression, anxiety and tension/stress. It will be assessed at baseline, postintervention and follow-up.

\section{PTSD symptoms}

The PCL-5 is a 20-item self-report measure that assesses the Diagnostic and Statistical Manual of Mental Disorders, Fifth Editio (DSM-5) symptoms of PTSD. ${ }^{40}$ Only the first responders will be administered this questionnaire, and it will be assessed at baseline, postintervention and follow-up.

\section{Sleep quality}

The Pittsburgh Sleep Quality Index will be used to assess the quality and patterns of sleep in the past month. ${ }^{41}$ This self-report questionnaire will be assessed at baseline, postintervention and follow-up.

\section{Quality of life}

The AQoL-6D quality of life questionnaire will be used to assess six separately scored dimensions to provide a simple global 'utility' score. ${ }^{42}$ Dimensions include pain, relationships, independent living, mental health, coping and senses. It will be assessed at baseline, postintervention and follow-up.

\section{Social support to exercise}

The social support and exercise survey will be used as an assessment of the level of support individuals making health behaviour changes (exercise) felt they were receiving from family and friends. ${ }^{43}$ It has been adapted for the purpose of this study to assess the past month instead of past 3 months. It will be assessed at weeks baseline, postintervention and follow-up.

\section{Suicidal ideation}

The SIDAS will be used to assess the presence and severity of suicidal thoughts. ${ }^{44}$ It consists of five items, each targeting an attribute of suicidal thoughts: frequency, controllability, closeness to attempt, level of distress and impact on daily functioning. It will be assessed at baseline, postintervention and follow-up.

\section{Service utilisation}

Mental health service utilisation will be assessed using a two-item questionnaire that asks participants whether they have commenced any additional mental health treatments during the intervention period (eg, medication changes and psychotherapy) and, if so, at what week of the intervention.

\section{Statistical analysis}

To examine the effect of the intervention on the primary outcome (K6), data from each iteration will be combined and an interrupted time series design will compare the rate of change in $\mathrm{K} 6$ on multiple occasions before intervention, to the rate of change during, and following the intervention period. A piecewise latent growth model clustered by partners and cohort will be fitted in MPlus to determine difference in slope between preintervention, during intervention and postintervention, controlling for baseline DASS, PCL and position (first responder or partner) and time varying covariates of weekly Fitbit steps and minutes of PA.

Outcomes measured at pre, post and follow-up will be entered into a multivariate mixed models analysis to control for type 1 error and account for clustering of individuals within each pair and cohort. This will test whether participants show a reduction in DASS, PCL symptoms and an increase in sleep quality and quality of life. Changes in symptom scores will also be regressed on changes in Fitbit PA levels to determine whether increases in PA levels are associated with reductions in symptoms and improvement in quality of life and sleep quality.

\section{Power analysis}

To determine the power required to detect a significant difference between baseline and intervention, we conducted Monte Carlo simulations based on the parameters observed in our pilot study, which included 10 pairs $(n=20)$. Two participants were excluded as outliers due to major adverse events not associated with the intervention. Monte Carlo simulations were conducted using Mplus 8. First, a piecewise latent growth curve model was fitted to the repeated fortnightly observations of the K6 pilot data controlling for baseline DASS score and position (first responder or partner) and clustered by partner pair. Model constraints tested for the difference between the slope during baseline and the slope during the intervention. The parameters from this model were entered into a Monte Carlo power simulation (10 000 sample draws), which showed that a sample size of 80 would be sufficient to achieve a power of $80 \%$. Five groups of $n=20$ will be recruited to account for the estimated dropout rate of less than $15 \%$ based on our pilot study.

\section{DISCUSSION}

This study has been designed to assess the efficacy of an online PA intervention delivered through Facebook. The study will employ novel strategies to reach firstnresponders and their support partners to assist them in engaging in regular PA. As the use of online technology is gaining popularity in mental healthcare and mobile technologies become increasingly available and affordable, adapting these methods for use in PA promotion will 
become increasingly important. ${ }^{45}$ This study makes use of four different forms of e-health technologies including: social media and telehealth to deliver education, wearable technologies and the smartphone application for data collection. These different forms of delivery offer a novel way to extend support outside of mental health settings in a way that is cost-effective and scalable.

Experience-based codesign of interventions within a mental health context is required so that service users and health professionals can work towards common goals to improve outcomes. ${ }^{46}$ The codesign of our PA intervention with the facilitators from Behind the Seen offers a promising approach to PA provision. Their inclusion in the design extends to their inclusion in the Facebook group, which is aimed to aid discussion in a way that is relatable to participants. Their feedback throughout the intervention on the delivery of content is also important.

As social support has been found to be a strong predictor of exercise adoption and maintenance, ${ }^{47}$ this study targets social isolation as a barrier to engagement by providing peer-to-peer support within the Facebook group and including support partners. Previous research has found that carers have the potential to support and extend interventions that are aimed at improving chronic disease risk behaviours of people with mental illness. ${ }^{48}$ However, there is currently limited information or services available to support family and carers in helping their family members, with carers reporting they often feel disconnected from healthcare professionals. ${ }^{49-51} \mathrm{We}$ specifically aim to address this gap by providing carers with the information and support needed for exercise adoption. Moreover, the impact of caregiving on 'informal' carers' own physical and mental health is also well established in the literature with carers noting they feel their own physical and mental health is neglected by the healthcare system. ${ }^{52-54}$ Therefore, this study will actively include support partners who have a close relationship to the first responder to help improve their physical and mental health as well.

A PA intervention is likely to be well accepted in this population as it is generally seen as non-stigmatising. This is particularly important because of the widely held perception that when accessing mental health services first responders do so at risk to their future career. ${ }^{55}$ The online platform therefore presents a unique opportunity to reach first responders who might struggle with access or engagement with mental healthcare and act as a facilitator to treatment. Our intervention may also provide an avenue to engage people in community-based PA initiatives such as sports groups and gyms. The results of this study will determine if an online exercise intervention can be successfully implemented among first responders.

\section{Limitations}

Our study has several potential limitations. The online recruitment pathway may provide a biased sample towards people who engage with social media and are technologically literate. We also anticipate that the weekly questionnaires will be burdensome. Finally, while we are trialling a novel stepped-wedged design to overcome the drawbacks of a traditional RCT, the limitation is that we cannot compare the results to an active control.

\section{Ethics and dissemination}

Informed consent will be obtained by the study investigator from all participants will be obtained prior to the baseline assessment.

The University of New South Wales in partnership with Behind the Seen will play a key role in establishing and achieving the knowledge translation goals given the differing expertise and focus. For example, this study will employ an integrated knowledge translation and exchange strategy designed to share research results with a wide variety of stakeholders. This project involves a partnership between researchers and a non-government organisation representing consumer and practitioner interest. The Knowledge Translation Planning Template will be used to identify specific audiences for the research and determine the best evidence regarding knowledge translation strategies to share research results. ${ }^{56}$ Our main knowledge users are first responders and their families, mental health clinicians and exercise professionals. Peer-reviewed publication(s) and academic conference presentations will reach the scientific community. All members of the investigative team will act as ambassadors of the study and communicate study outcomes via their organisational and personal websites and social media links. For example, Behind The Seen has a strong social media presence and following among first responders and therefore a potential avenue by which interest and awareness can be generated, regarding community PA programmes and gyms. Educational outreach through Behind The Seen's extensive network are also strategies that will be used. This also provides an avenue by which research findings can be disseminated to relevant consumer groups in a timely manner. Research dissemination and knowledge translation to occur through formal conferences and training, and the dissemination of educational materials. Given we are not restricted by geographical location, this project offers a unique opportunity to work with Australian-wide community facilities to facilitate knowledge translation and increase uptake of services.

\section{Author affiliations}

${ }^{1}$ School of Psychiatry, UNSW, Sydney, New South Wales, Australia

${ }^{2}$ St John of God Health Care North Richmond Hospital North, North Richmond, New South Wales, Australia

${ }^{3}$ Black Dog Institute, Prince of Wales Hospital, Sydney, New South Wales, Australia ${ }^{4}$ School of Psychology, University of New South Wales, Sydney, New South Wales, Australia

${ }^{5}$ University Psychiatric Centre KU Leuven, Leuven, Belgium

${ }^{6}$ Department of Rehabilitation Sciences, KU Leuven, Leuven, Vlaanderen, Belgium

Acknowledgements The authors would like to acknowledge the contribution of Veronique Moseley and Ross Beckley from Behind the Seen Australia.

Contributors GM and SR conceived the study. GM drafted the protocol with input from ZS, RW, JMN and DV. DH-P, ZS and RW provided statistical expertise. All 
authors contributed to the study design. GM and SR will run the online Facebook group with input from RW, JMN and DV. DHP will lead the statistical analysis. All authors critically reviewed the manuscript.

Funding The authors have not declared a specific grant for this research from any funding agency in the public, commercial or not-for-profit sectors.

Competing interests None declared.

Patient consent for publication Not required.

Provenance and peer review Not commissioned; externally peer reviewed.

Open access This is an open access article distributed in accordance with the Creative Commons Attribution Non Commercial (CC BY-NC 4.0) license, which permits others to distribute, remix, adapt, build upon this work non-commercially, and license their derivative works on different terms, provided the original work is properly cited, appropriate credit is given, any changes made indicated, and the use is non-commercial. See: http://creativecommons.org/licenses/by-nc/4.0/.

\section{REFERENCES}

1. Harvey SB, Milligan-Saville JS, Paterson HM, et al. The mental health of fire-fighters: an examination of the impact of repeated trauma exposure. Aust N Z J Psychiatry 2016;50:649-58.

2. Harvey S, Devilly G, Forbes D, et al. Expert guidelines: diagnosis and treatment of post-traumatic stress disorder in emergency service workers 2015.

3. Fullerton CS, Ursano RJ, Wang L. Acute stress disorder, posttraumatic stress disorder, and depression in disaster or rescue workers. Am J Psychiatry 2004;161:1370-6.

4. Berger W, Coutinho ESF, Figueira I, et al. Rescuers at risk: a systematic review and meta-regression analysis of the worldwide current prevalence and correlates of PTSD in rescue workers. Soc Psychiatry Psychiatr Epidemiol 2012;47:1001-11.

5. Creamer M, Burgess P, McFarlane AC. Post-Traumatic stress disorder: findings from the Australian national survey of mental health and well-being. Psychol Med 2001;31:1237-47.

6. Smith TC, Ryan MAK, Wingard DL, et al. New onset and persistent symptoms of post-traumatic stress disorder self reported after deployment and combat exposures: prospective population based US military cohort study. BMJ 2008;336:366-71.

7. Paul Barratt LS, Palmer M. When helping hurts: PTSD in Firstresponders: Australia21; 2018.

8. McLaughlin KA, Koenen KC, Friedman MJ, et al. Subthreshold posttraumatic stress disorder in the world Health organization world mental health surveys. Biol Psychiatry 2015;77:375-84.

9. Momartin S, Silove D, Manicavasagar V, et al. Comorbidity of PTSD and depression: associations with trauma exposure, symptom severity and functional impairment in Bosnian refugees resettled in Australia. J Affect Disord 2004;80:231-8.

10. Schnurr PP, Lunney CA, Sengupta A, et al. A longitudinal study of retirement in older male veterans. J Consult Clin Psychol 2005;73:561-6.

11. Jakupcak M, Cook J, Imel Z, et al. Posttraumatic stress disorder as a risk factor for suicidal ideation in Iraq and Afghanistan war veterans. J Trauma Stress 2009;22:303-6.

12. Roberts AL, Agnew-Blais JC, Spiegelman D, et al. Posttraumatic stress disorder and incidence of type 2 diabetes mellitus in a sample of women: a 22-year longitudinal study. JAMA Psychiatry 2015;72:203-10.

13. Bartoli F, Crocamo C, Alamia A, et al. Posttraumatic stress disorder and risk of obesity: systematic review and meta-analysis. J Clin Psychiatry 2015;76:e1253-61.

14. Rosenbaum S, Stubbs B, Ward PB, et al. The prevalence and risk of metabolic syndrome and its components among people with posttraumatic stress disorder: a systematic review and metaanalysis. Metabolism 2015;64:926-33.

15. Boscarino JA. Posttraumatic stress disorder and physical illness: results from clinical and epidemiologic studies. Ann N Y Acad Sci 2004:1032:141-53.

16. Vancampfort D, Richards J, Stubbs B, et al. Physical activity in people with PTSD: a systematic review of correlates. J Phys Act Health 2016;13:910-8.

17. Vancampfort D, Knapen J, Probst M, et al. A systematic review of correlates of physical activity in patients with schizophrenia. Acta Psychiatr Scand 2012;125:352-62.

18. de Assis MA, de Mello MF, Scorza FA, et al. Evaluation of physical activity habits in patients with posttraumatic stress disorder. Clinics 2008;63:473-8.
19. Stubbs B, Vancampfort D, Hallgren M, et al. EPA guidance on physical activity as a treatment for severe mental illness: a metareview of the evidence and position statement from the European psychiatric association (EPA), supported by the International organization of physical therapists in mental health (IOPTMH). Eur Psychiatry 2018;54:124-44

20. Rosenbaum S, Vancampfort D, Steel Z, et al. Physical activity in the treatment of post-traumatic stress disorder: a systematic review and meta-analysis. Psychiatry Res 2015;230:130-6.

21. Rosenbaum S, Tiedemann A, Sherrington C, et al. Physical activity interventions for people with mental illness: a systematic review and meta-analysis. J Clin Psychiatry 2014;75:964-74.

22. Haugen PT, McCrillis AM, Smid GE, et al. Mental health stigma and barriers to mental health care for first responders: a systematic review and meta-analysis. J Psychiatr Res 2017;94:218-29.

23. Schuch FB, Vancampfort D, Firth J, et al. Physical activity and incident depression: a meta-analysis of prospective cohort studies. Am J Psychiatry 2018;175:631-48.

24. Brown RA, Prince MA, Minami H, et al. An exploratory analysis of changes in mood, anxiety and craving from pre- to post-single sessions of exercise, over 12 weeks, among patients with alcohol dependence. Ment Health Phys Act 2016;11:1-6.

25. Rosenbaum S, Sherrington $C$, Tiedemann A. Exercise augmentation compared with usual care for post-traumatic stress disorder: a randomized controlled trial. Acta Psychiatr Scand 2015;131:350-9.

26. Harvey SB, Øverland S, Hatch SL, et al. Exercise and the prevention of depression: results of the HUNT cohort study. Am J Psychiatry 2018;175:28-36.

27. Cavallo DN, Tate DF, Ries AV, et al. A social media-based physical activity intervention: a randomized controlled trial. Am J Prev Med 2012;43:527-32.

28. Naslund JA, Aschbrenner KA, Marsch LA, et al. Facebook for supporting a lifestyle intervention for people with major depressive disorder, bipolar disorder, and schizophrenia: an exploratory study. Psychiatr Q 2018;89:81-94.

29. Moran J, Kelly G, Haberlin C, et al. The use of eHealth to promote physical activity in patients with mental health conditions: a systematic review. HRB Open Res 2018;1.

30. Wheeler AJ, Roennfeldt H, Slattery M, et al. Codesigned recommendations for increasing engagement in structured physical activity for people with serious mental health problems in Australia. Health Soc Care Community 2018;26:860-70.

31. Soundy A, Freeman P, Stubbs B, et al. The value of social support to encourage people with schizophrenia to engage in physical activity: an international insight from specialist mental health physiotherapists. J Ment Health 2014;23:256-60.

32. Schuch FB, Dunn AL, Kanitz AC, et al. Moderators of response in exercise treatment for depression: a systematic review. J Affect Disord 2016;195:40-9.

33. Norton KCJ, Parker R, Williams A, et al. New Australian standard for adult pre-exercise screening. Sport Health 2012;30:12-18.

34. Kessler RC, Andrews G, Colpe LJ, et al. Short screening scales to monitor population prevalences and trends in non-specific psychological distress. Psychol Med 2002;32:959-76.

35. Naslund JA, Aschbrenner KA, Marsch LA, et al. Feasibility and acceptability of Facebook for health promotion among people with serious mental illness. Digit Health 2016;2. doi:10.1177/2055207616654822

36. Braun V, Clarke V. Using thematic analysis in psychology. Qual Res Psychol 2006;3:77-101.

37. Greenwood JLJ, Joy EA, Stanford JB. The physical activity vital sign: a primary care tool to guide counseling for obesity. J Phys Act Health 2010;7:571-6.

38. Rosenbaum S, Ward PB, International Working Group. The simple physical activity questionnaire. Lancet Psychiatry 2016;3:e1.

39. Henry JD, Crawford JR. The short-form version of the depression anxiety stress scales (DASS-21): construct validity and normative data in a large non-clinical sample. Br J Clin Psychol 2005;44:227-39.

40. Blevins CA, Weathers FW, Davis MT, et al. The Posttraumatic Stress Disorder Checklist for DSM-5 (PCL-5): Development and Initial Psychometric Evaluation. J Trauma Stress 2015;28:489-98.

41. Buysse DJ, Reynolds CF, Monk TH, et al. The Pittsburgh sleep quality index: a new instrument for psychiatric practice and research. Psychiatry Res 1989;28:193-213.

42. Richardson JRJ, Peacock SJ, Hawthorne G, et al. Construction of the descriptive system for the assessment of quality of life AQoL-6D utility instrument. Health Qual Life Outcomes 2012;10:38.

43. Sallis JF, Grossman RM, Pinski RB, et al. The development of scales to measure social support for diet and exercise behaviors. Prev Med 1987;16:825-36. 
44. van Spijker BAJ, Batterham PJ, Calear AL, et al. The suicidal ideation attributes scale (SIDAS): community-based validation study of a new scale for the measurement of suicidal ideation. Suicide Life Threat Behav 2014:44:408-19.

45. Firth J, Torous J, Yung AR. Ecological momentary assessment and beyond: the rising interest in e-mental health research. $J$ Psychiatr Res 2016;80:3-4

46. Matthews E, Cowman M, Denieffe S. Using experience-based co-design for the development of physical activity provision in rehabilitation and recovery mental health care. J Psychiatr Ment Health Nurs 2017;24:545-52.

47. Firth J, Rosenbaum S, Stubbs B, et al. Motivating factors and barriers towards exercise in severe mental illness: a systematic review and meta-analysis. Psychol Med 2016;46:2869-81.

48. Aschbrenner KA, Mueser KT, Naslund JA, et al. Facilitating partner support for lifestyle change among adults with serious mental illness: a feasibility pilot study. Community Ment Health $J$ 2017;53:394-404.

49. Bailey JM, Wye PM, Wiggers $\mathrm{JH}$, et al. Family carers: a role in addressing chronic disease risk behaviours for people with a mental illness? Prev Med Rep 2017;7:140-6.
50. Albert R, Simpson A. Double deprivation: a phenomenological study into the experience of being a carer during a mental health crisis. $J$ Adv Nurs 2015;71:2753-62.

51. ROWE J. Great expectations: a systematic review of the literature on the role of family carers in severe mental illness, and their relationships and engagement with professionals. J Psychiatr Ment Health Nurs 2012;19:70-82.

52. Gutiérrez-Maldonado J, Caqueo-Urízar A, Kavanagh DJ. Burden of care and general health in families of patients with schizophrenia. Soc Psychiatry Psychiatr Epidemiol 2005;40:899-904.

53. Savage $S$, Bailey $S$. The impact of caring on caregivers' mental health: a review of the literature. Aust Health Rev 2004;27:111-7.

54. Happell B, Wilson K, Platania-Phung C, et al. Physical health and mental illness: listening to the voice of carers. J Ment Health 2017;26:127-33.

55. Mick Palmer BW. A First Responders' Perspective. Trauma - related stress in Australia. Essays by leading Australian thinkers and researchers 2016:38-43.

56. Barwick M. Knowledge translation planning template. Ontario: The Hospital for Sick Children, 2013. 